\title{
Upregulation of Gene Expression in Reward-Modulatory Striatal Opioid Systems by Sleep Loss
}

\author{
Brian A Baldo*, ,, Erin C Hanlon ${ }^{3,4}$, William Obermeyer ${ }^{1,4}$, Quentin Bremer', Elliott Paletz' and \\ Ruth M Benca ${ }^{1,2}$
}

'Department of Psychiatry, University of Wisconsin-Madison, School of Medicine and Public Health, Madison, WI, USA; ${ }^{2}$ Neuroscience Training Program, University of Wisconsin-Madison, School of Medicine and Public Health, Madison, WI, USA; ${ }^{3}$ Section on Endocrinology, Diabetes, and Metabolism, Department of Medicine, University of Chicago School of Medicine, Chicago, IL, USA

\begin{abstract}
Epidemiological studies have shown a link between sleep loss and the obesity 'epidemic,' and several observations indicate that sleep curtailment engenders positive energy balance via increased palatable-food 'snacking.' These effects suggest alterations in rewardmodulatory brain systems. We explored the effects of 10 days of sleep deprivation in rats on the expression of striatal opioid peptide (OP) genes that subserve food motivation and hedonic reward, and compared effects with those seen in hypothalamic energy balanceregulatory systems. Sleep-deprived (Sleep-Dep) rats were compared with yoked forced-locomotion apparatus controls (App-Controls), food-restricted rats (Food-Restrict), and unmanipulated controls (Home-Cage). Detection of mRNA levels with in situ hybridization revealed a subregion-specific upregulation of striatal preproenkephalin and prodynorhin gene expression in the Sleep-Dep group relative to all other groups. Neuropeptide Y (NPY) gene expression in the hippocampal dentate gyrus and throughout neocortex was also robustly upregulated selectively in the Sleep-Dep group. In contrast, parallel gene expression changes were observed in the Sleep-Dep and Food-Restrict groups in hypothalamic energy-sensing systems (arcuate nucleus NPY was upregulated, and cocaine- and amphetamine-regulated transcript was downregulated), in alignment with leptin suppression in both groups. Together, these results reveal a novel set of sleep deprivation-induced transcriptional changes in reward-modulatory peptide systems, which are dissociable from the energy-balance perturbations of sleep loss or the potentially stressful effects of the forced-locomotion procedure. The recruitment of telencephalic food-reward systems may provide a feeding drive highly resistant to feedback control, which could engender obesity through the enhancement of palatable feeding.

Neuropsychopharmacology (2013) 38, 2578-2587; doi:I0.1038/npp.2013.174; published online 21 August 2013
\end{abstract}

Keywords: sleep loss; food reward; energy homeostasis; striatum; nucleus accumbens; opioid peptide

\section{INTRODUCTION}

The escalating social trend toward shortened average sleep duration may be contributing significantly to the obesity epidemic (Van Cauter and Knutson, 2008). Decreased sleep in humans is associated with weight gain, higher body mass index (BMI), greater body-fat percentage (Cappuccio et al, 2008; Patel and $\mathrm{Hu}, 2008$ ), and impaired glucose metabolism (Hanlon and Van Cauter, 2011; Nedeltcheva et al, 2009). Remarkably, recent studies have shown that the positive energy-balance state incurred by sleep loss is accounted for by increased caloric intake outside of regular meals ('snacking'; Markwald et al, 2013), particularly on carbohydrate- or fat-enriched foods (Markwald et al, 2013; Nedeltcheva et al, 2009; Spiegel et al, 2004a; St-Onge et al,

*Correspondence: Dr BA Baldo, Department of Psychiatry, University of Wisconsin-Madison, School of Medicine and Public Health, 6001 Research Park Boulevard, Madison, WI 53719, USA, Tel: + 608263 4019, Fax: + | 608265 3050, E-mail: babaldo@wisc.edu ${ }^{4}$ These authors contributed equally to this work.

Received 18 June 2013; revised I July 2013; accepted 5 July 2013; accepted article preview online 18 July 2013
2011; Weiss et al, 2010). There is much interest in identifying the physiological basis of these behavioral changes, and studies to date have focused mainly on peripheral endocrine systems (eg, leptin and ghrelin) that regulate feeding in conjunction with energy-balance fluctuations. In some human studies, leptin and ghrelin levels are altered in sleep loss in directions that promote food intake, and rodent studies have shown that sleep curtailment modulates hypothalamic peptides and other systems whose activity tracks these circulating hormones (Barf et al, 2012; Koban et al, 2006; Martins et al, 2010; Spiegel et al, 2004b).

Nevertheless, a recent study in humans showed that increased snacking in sleep loss could not be explained by changes in peripheral hunger or satiety hormones alone (Markwald et al, 2013), implicating systems that drive 'nonhomeostatic' feeding; that is, excess feeding driven by motivational or cognitive processes. To date, however, the effects of sleep loss on neuromodulators governing the hedonic aspects of feeding, such as the opioid peptide (OP), enkephalin (ENK), are unknown. Stimulation of receptors for ENK within striatal subregions (particularly the nucleus 
accumbens (Acb)) augments the 'liking' of sweet tastes (Pecina and Berridge, 2005) and enhances the intake of sweet, and/or fat-rich foodstuffs (Woolley et al, 2006; Zhang et al, 1998)-the same types of food whose intake is augmented in sleep loss. Furthermore, endogenous ENK release in rat striatum accompanies the 'binge-like' intake of palatable food (Difeliceantonio et al, 2012), and striatal ENK gene expression is upregulated by palatable food-conditioned cues (Schiltz et al, 2007). ENK and related systems could therefore have an important role in the palatable or high-fat food snacking seen with sleep loss in humans, which appears to be a crucial factor promoting weight gain (Markwald et al, 2013; StOnge et al, 2011).

Here, we investigated the effects of sleep deprivation in rats on gene expression in several feeding-related CNS peptide systems, chosen to provide points of contrast between telencephalic reward-modulatory substrates (including striatal OPs) and hypothalamic systems responsive to energy-balance status. Sleep deprivation was achieved using forced locomotion on a moving belt, automatically triggered during sleep episodes (Newman et al, 2009). We used two controls: a group to control for the sleep deprivation stimulus, in which rats were pair-yoked to sleep-deprived (Sleep-Dep) counterparts, with forcedlocomotion epochs 'played back' during waking; and a food-restricted (Food-Restrict) group in which daily food allotments were calibrated to precisely match body weight changes of Sleep-Dep counterparts. The latter was done to tease apart effects of sleep loss from secondary effects arising from weight loss, which is commonly seen in rodent models of sleep deprivation (Newman et al, 2009; Rechtschaffen and Bergmann, 1995). We show a significant transcriptional upregulation of OPs in reward-related striatal regions following sleep loss, relative to both control manipulations. The implications of these findings for understanding reward-driven 'snacking' seen in sleeprestricted humans are discussed below.

\section{MATERIALS AND METHODS}

\section{Subjects}

Male Sprague-Dawley rats (Harlan Laboratories, Madison, WI) weighing 300-325 g were housed in pairs in clear cages with ad libitum access to food and water (except for certain experiments as described below) in a light- and temperature-controlled vivarium. They were maintained under a 12-h:12-h light-dark cycle (lights on at 0700 hours). All facilities and procedures were in accordance with the guidelines regarding animal use and care from the US National Institutes of Health, and were supervised and approved by the Institutional Animal Care and Use Committee of the University of Wisconsin.

\section{Conveyer-Over-Water (COW) Apparatus and Apparatus Control Procedure}

The COW apparatus used here is similar in principle to the disk-over-water procedure, but uses motion detection rather than online EEG recording to trigger wake-inducing belt movements. Motion detection sleep deprivation with the COW previously been validated with EEG recording of sleep (Newman et al, 2009). A photograph of the COW apparatus is shown in Supplementary Figure S1 of Supplementary Information, and a detailed description is provided in Supplementary Materials and Methods. For apparatus control (App-Control) rats, the belt movements were played back from those recorded from a matched sleep-restricted animal, but the belt moved only when the control animal was awake. To minimize sleep disturbance in control rats, yet subject them to the same amount of belt rotation, the speed of the belt was speeded up by $50 \%$ and inter-stimuli intervals were decreased by one-third. Rats in the COWs were videotaped, and visual scoring of behavior was carried out daily on samples of video recording to ensure validity of motion detection-based sleep deprivation.

\section{Food Restriction Procedure}

Daily food allotment was adjusted to maintain a similar rate of weight loss as seen in the sleep-restricted rats. Rats in the Food-Restrict groups were housed in their home cages and never exposed to the COWs. The Food-Restrict and sleep-restricted groups were matched with regard to age and initial body weight. Rats' daily food allotments were adjusted so that the percentage of daily body weight loss matched the previous day's weight loss of the Sleep-Dep counterpart (see Supplementary Materials and Methods for further details).

\section{Experimental Design}

Two distinct experiments were carried out. Experiment 1 compared Sleep-Dep rats ('Sleep-Dep' group; $N=11$ ) with Food-Restrict rats ('Food-Restrict' group; $N=11$ ) that lost the same amount of weight. Experiment 2 compared SleepDep rats $(N=15)$ with forced-locomotion apparatus controls ('App-Controls' group; $N=15$ ). Home cage-housed rats ('Home-Cage' group) were included as controls in both experiments $(N=11$ for experiment $1 ; N=17$ for experiment 2). In experiment 1 , to maximize chances of a successful outcome, we used two 10-day sleep deprivation periods, with an in-between 10-day recovery period, based on the laboratory's past experience with such a protocol. In experiment 2, however, only one period of sleep deprivation was used because in experiment 1 , we found this to be sufficient to robustly elevate feeding and we wanted to minimize the risk of subject drop-out because of mechanical problems with the COWs. We found no substantial differences between the two experiments with regard to the genes under study.

On the final day of the 10-day experimental period, rats of all groups were killed by decapitation under isoflurane anesthesia. Decapitations occurred between 1030 and 1200 hours; this was consistent across all groups for experiments 1 and 2 . Their brains were rapidly dissected, flash frozen in $-20{ }^{\circ} \mathrm{C}$ 2-methylbutane, and stored at $-80^{\circ} \mathrm{C}$ until cryostat sectioning. Trunk blood was also collected and processed at the time of killing; radioimmunoassays for plasma leptin and insulin were conducted according to standard techniques, as described in Supplementary Materials and Methods. 


\section{In Situ Hybridization}

Brain slices were subjected to processing for in situ hybridization, according to standard methods (details are given in the Supplementary Materials and methods, including primers for probe generation in Supplementary Table S1). After processing, sections were exposed to a phosphorimager screen for 1-14 days, depending on intensity of the signal. The screen was scanned on a Typhoon scanner and quantification of average optical density in particular brain regions was performed using ImageQuant 5.2 software (Molecular Dynamics). Quantification of signal was standardized across rats using predetermined fixed-area shapes for each region of interest (ROI). Striatal ROIs are shown in Supplementary Figure S2. ROIs for neuropeptide Y (NPY) and cocaine- and amphetamine-regulated transcript (CART) are depicted in Figure 4.

\section{Statistical Analyses}

For the in situ hybridization data, the averaged pixel intensities for each region were analyzed by first performing a global two-factor mixed-design ANOVA (group $\times$ brain region). Following significance in this ANOVA, comparisons among the groups for each individual brain region was performed using one-factor (group) between-subjects ANOVAs.

\section{RESULTS}

\section{Effects on OP Gene Expression in the Striatum}

As shown in Figures 1 and 2, we observed a robust upregulation in proenkephalin (ENK) and prodynorphin (DYN) peptide gene expression in multiple striatal subregions in the Sleep-Dep group relative to the other groups (group $\times$ subregion interactions for ENK, experiment 1: $\mathrm{F}(12,203)=10.2, \quad P<0.001$, ENK, experiment 2: $\quad \mathrm{F}(12,308)=3.7, \quad P<0.001 ; \quad \mathrm{DYN}, \quad$ experiment 1 : $\mathrm{F}(12,175)=1.9, \quad P=0.03$, experiment $2: \mathrm{F}(12,308)=6.2$; $P<0.001)$. Based on these results, subanalyses by striatal subregion for each gene separately were carried out and summarized in Supplementary Table S2. The most common effect observed was an increase in opioid precursor peptide mRNA levels in the Sleep-Dep group relative to all other groups, and the strong elevations in opioid gene expression seen in experiment 1 were replicated in experiment 2. The Acb core showed the strongest and most selective differentiation between the Sleep-Dep $v s$ other groups both ENK and DYN, with increases in the Sleep-Dep group $v s$ no effect in either the App-Control or Food-Restrict groups. Furthermore, the medial Acb shell, a region strongly implicated in $\mu$-opioid-dependent reward modulation (Pecina and Berridge, 2005), displayed a highly selective upregulation of ENK expression in the Sleep-Dep group relative to the Food-Dep group. There was a small but significant increase in DYN in the medial Acb shell of AppControls. Outside the Acb, the most common pattern was, again, for opioid gene expression to be significantly higher in the Sleep-Dep group relative to other groups. There were small but significant elevations of DYN in the ventrolateral and dorsal striatum of App-Control relative to Home-Cage rats; however, DYN upregulation was significantly greater in Sleep-Dep vs App-Control rats for these regions (see Supplementary Table S2).

The dissociation between weight loss $v s$ sleep loss can be appreciated in the scatterplots of Figure 3. Within the same range of weight loss, the distribution of optical density values (the index of gene expression levels), particularly for ENK, is largely non-overlapping for the Food-Restrict and Sleep-Dep groups; moreover, it can be seen that the FoodRestrict group completely overlaps the Home-Cage group.

Neither ENK nor DYN expression was changed in any group in a control site, primary somatosensory cortex. Moreover, $c$-fos expression was not changed in any striatal area except DLS, where there was a small suppression in the App-Control group relative to the Sleep-Dep group $(\mathrm{F}(3,66)=3.1, P<0.04)$. This observation indicates that the upregulation of ENK and DYN was not the nonspecific outcome of a global upregulation of all striatal gene expression. Nor were striatal opioid effects a reflection of generalized upregulation in all central feeding-modulatory systems, because expression of hypothalamic melanin concentrating hormone $(\mathrm{MCH})$ was not changed in any group, and effects on hypocretin/orexin (H/O) were inconsistent: an upregulation in the Sleep-Dep group of experiment $1(\mathrm{~F}(2,25)=3.9 ; P<0.04)$, but not experiment 2 $(\mathrm{F}(2,44)=1.6 ; \mathrm{NS}) . \mathrm{MCH}$ and $\mathrm{H} / \mathrm{O}$ results are summarized in Supplementary Table S3.

\section{Effects on NPY and CART Expression}

As shown in Figure 4, sleep deprivation markedly augmented levels of mRNA for the NPY gene and suppressed levels for CART; these effects varied by anatomical region. For NPY, we analyzed the (1) the arcuate nucleus, (2) a sector of somatosensory cortex representative of all neocortex, and (3) dentate gyrus of the hippocampus where we noted a strikingly circumscribed signal in the Sleep-Dep rats (Figure 4, and Figure 3 of Supplementary Information). For NPY, there was a highly significant group $\times$ brain region interaction for experiment $1(\mathrm{~F}(4,87)=16.7 ; P<0.001)$ and experiment $2(\mathrm{~F}(4,126)=6.4 ; P<0.001)$. There were also significant effects for CART in an omnibus ANOVA, which incorporated the two brain regions in which CART was assayed, the arcuate nucleus and a hypothalamic ROI dorsal to the arcuate termed 'dorsal hypothalamus' for simplicity (group $\times$ brain region in experiment 1 : $\mathrm{F}(2,50)=18.3 ; \quad P<0.001 ;$ in experiment $2: \mathrm{F}(2,74)=4.8$; $P=0.01)$.

Based on these interactions and multiple strong main effects, we conducted further analyses with one-way ANOVAs in the individual brain regions. Effects can be seen in the digital images of Figure 4, and are summarized in Supplementary Table S4. In the arcuate nucleus, sleep deprivation and food restriction (but not the App-Control manipulation) robustly upregulated NPY expression and downregulated CART expression. For CART, sleep lossinduced downregulation was also seen in dorsal hypothalamus. These results agree with literature indicating that hypothalamic NPY and CART are regulated in a reciprocal manner in negative energy balance (Hillebrand et al, 2002).

Levels of NPY mRNA in the dentate gyrus (Figure 4, Supplementary Figure S3) and in neocortex (the ROI was an 
EXPERIMENT 1:
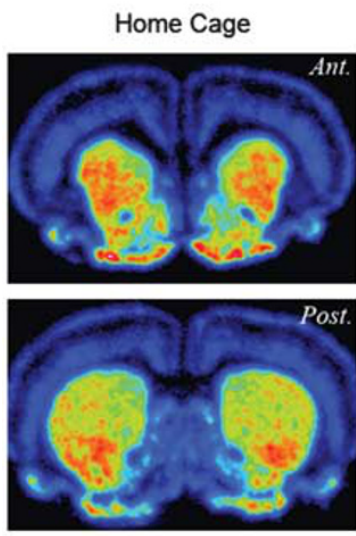

EXPERIMENT 2:
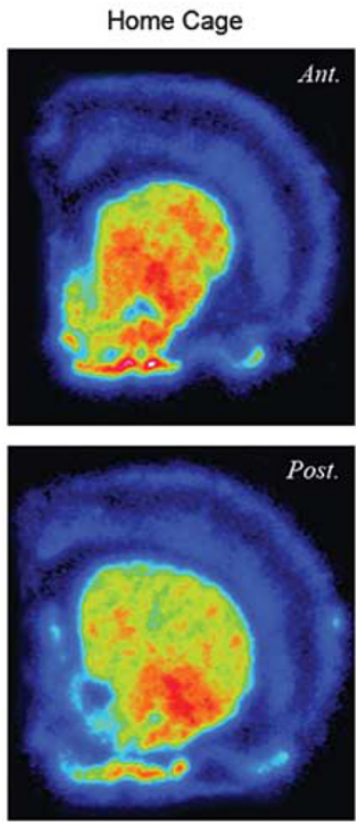

\section{Proenkephalin}
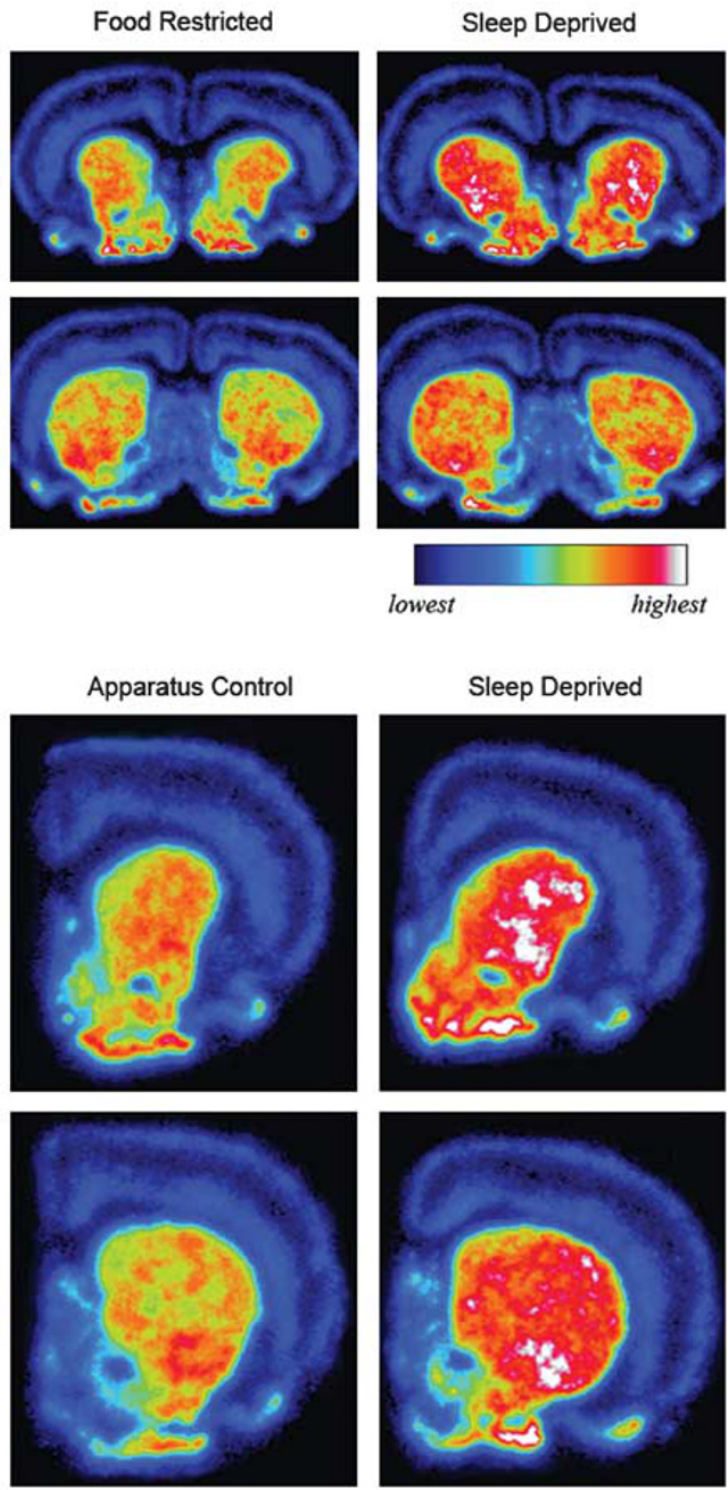

Figure I Pseudocolor images of coronal sections through two levels of the striatum (Ant., anterior; Post., posterior) from representative rats in each treatment group for each of the two experiments, showing proenkephalin expression. Color key depicts intensity range of labeling.

area in somatosensory cortex, but the effect was seen throughout neocortex) were upregulated in Sleep-Dep rats (dentate gyrus, experiment 1: $\mathrm{F}(2,30)=29.6 ; P<0.001$; experiment 2: $\mathrm{F}(3,65)=31.6 ; P<0.001)$. For the neocortex, the effect was significant in experiment $2(\mathrm{~F}(3,65)=30.9$; $P<0.001)$, but missed significance in experiment 1 $(\mathrm{F}(2,29)=2.2, \mathrm{NS})$. These effects were completely restricted to the Sleep-Dep rats; no effects on NPY expression were seen in other groups.

Figure 5 shows scatterplots of gene expression $v s$ weight loss in all groups for arcuate-localized NPY and hippocampal NPY. Arcuate nucleus gene expression levels are completely overlapping for the Food-Restrict and Sleep-Dep groups, but hippocampal gene expression values are completely non-overlapping, despite comparable weight loss in the two groups.

\section{Sleep}

Sleep epochs were reduced by $>85 \%$ (see Supplementary Figure S4) during the 10-day sleep deprivation periods. There was little change in the percentage of sleep epochs for the App-Control rats. Accuracy of the machine scoring approached $90 \%$ relative to the human reviewers.

Changes in Food Intake, Body Weight, Plasma Insulin, and Leptin

Sleep-Dep and Food-Restrict rats exhibited almost identical degrees of mean daily weight loss throughout the 10-day experimental periods of experiment 1 ; mean daily weight change (g/day \pm SEM) was $-3.7 \pm 0.2$ for the Sleep-Dep group, and $-3.9 \pm 0.2$ for the Food-Restrict group. The 


\section{Prodynorphin}

EXPERIMENT 1:

Home Cage
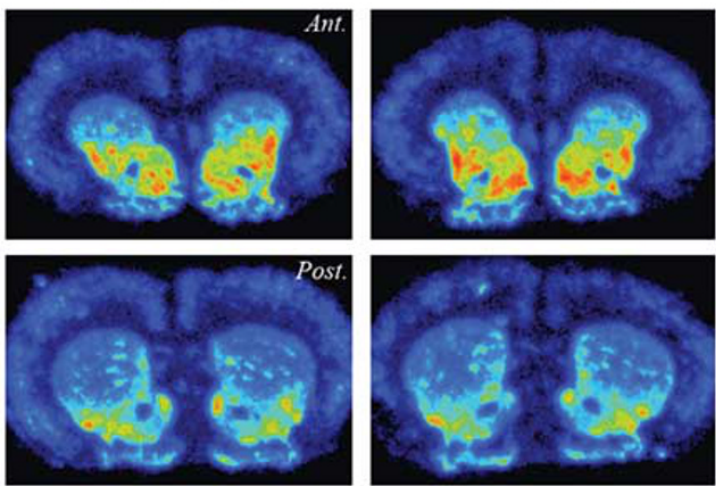

Sleep Deprived
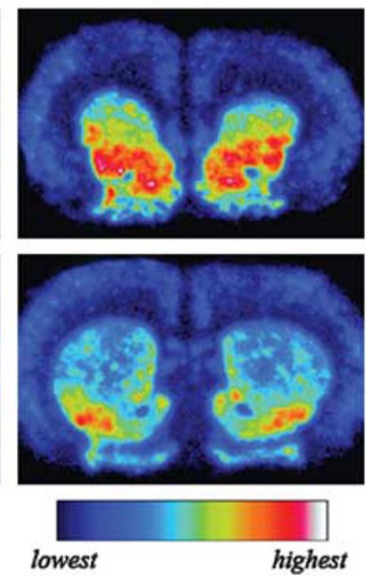

EXPERIMENT 2:

Home Cage
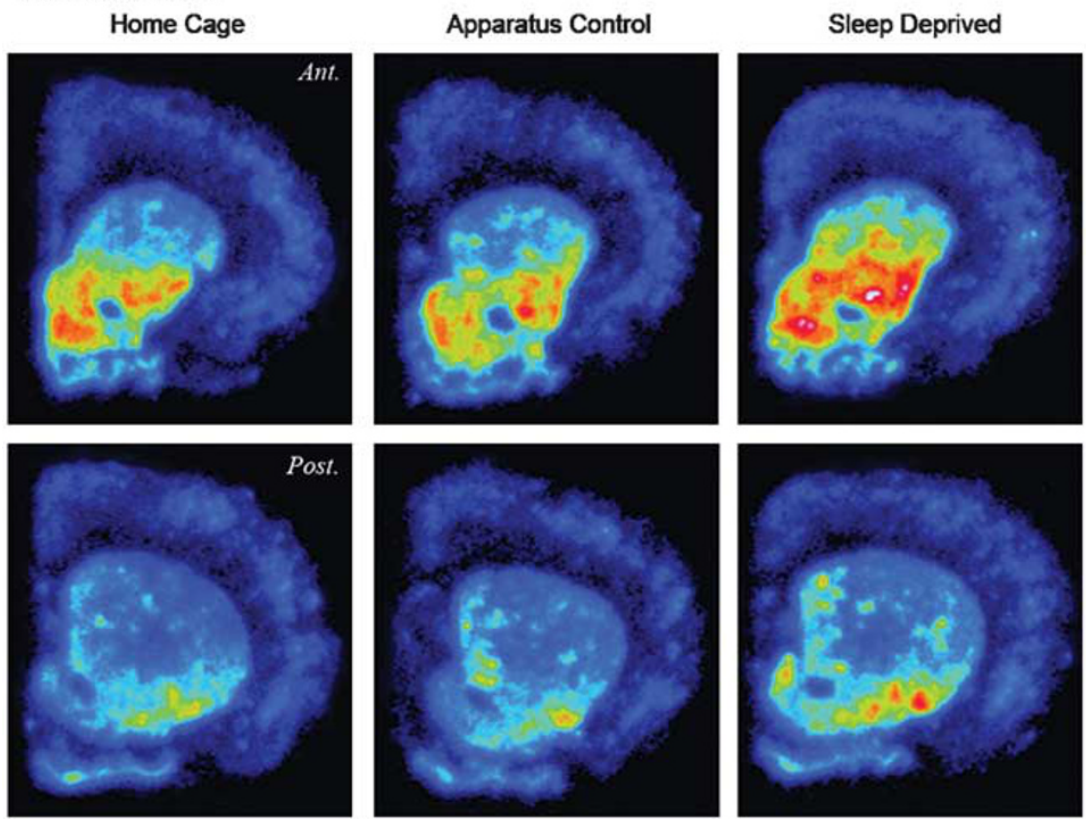

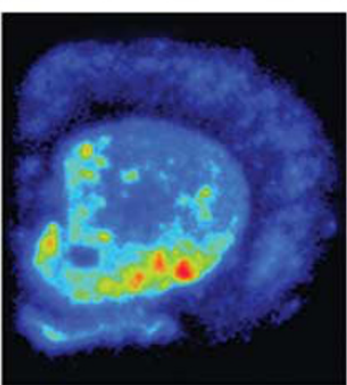

Figure 2 Pseudocolor images of coronal sections through two levels of the striatum (Ant., anterior; Post., posterior) from representative rats in each treatment group for each of the two experiments, showing prodynorphin expression. Color key depicts intensity range of labeling.

Sleep-Dep group of experiment 2 rats lost weight at about the same rate $(-2.85 \pm 0.2)$ as seen in experiment 1 . App-Control rats gained weight throughout the 10-day experimental period $(0.70 \pm 0.2)$ at about the same rate as Home-Cage controls of experiment $1(0.92 \pm 0.2)$, but at a lesser rate than Home-cage controls of experiment 2 $(2.0 \pm 0.2)$. Furthermore, as shown previously (eg, Newman et al, 2009; Rechtschaffen and Bergmann, 1995), food intake escalated over the days of sleep deprivation (see Supplementary Figure S5).

Confirming prior results (Barf et al, 2012; Spiegel et al, 2004a, b), leptin levels were depressed in the Sleep-Dep rats, beyond the leptin suppression seen in Food-Restrict rats or the mild suppression seen in App-Controls. Insulin was also suppressed in the Sleep-Dep groups (experiment 1: insulin, $\mathrm{F}(2,29)=8.3, P=0.001$; leptin, $\mathrm{F}(2,29)=29.4, P<0.001)$; experiment 2: insulin, $\mathrm{F}(2,44)=6.7, P=0.0028$; leptin, $\mathrm{F}(2,44)=51.4, P<0.0001$; see Supplementary Figure S6).

\section{DISCUSSION}

\section{Summary}

In this study, we found robust, sleep loss-associated transcriptional upregulation of telencephalic peptides that modulate higher-order motivation or learning processesspecifically, ENK and DYN throughout widespread areas of the striatum, and NPY in hippocampus. On an anatomical subregion basis, these effects were separable from weight loss or generalized perturbations of either hunger or the forced-locomotion sleep deprivation procedure. In contrast, in energy balance-regulatory systems of the hypothalamic 

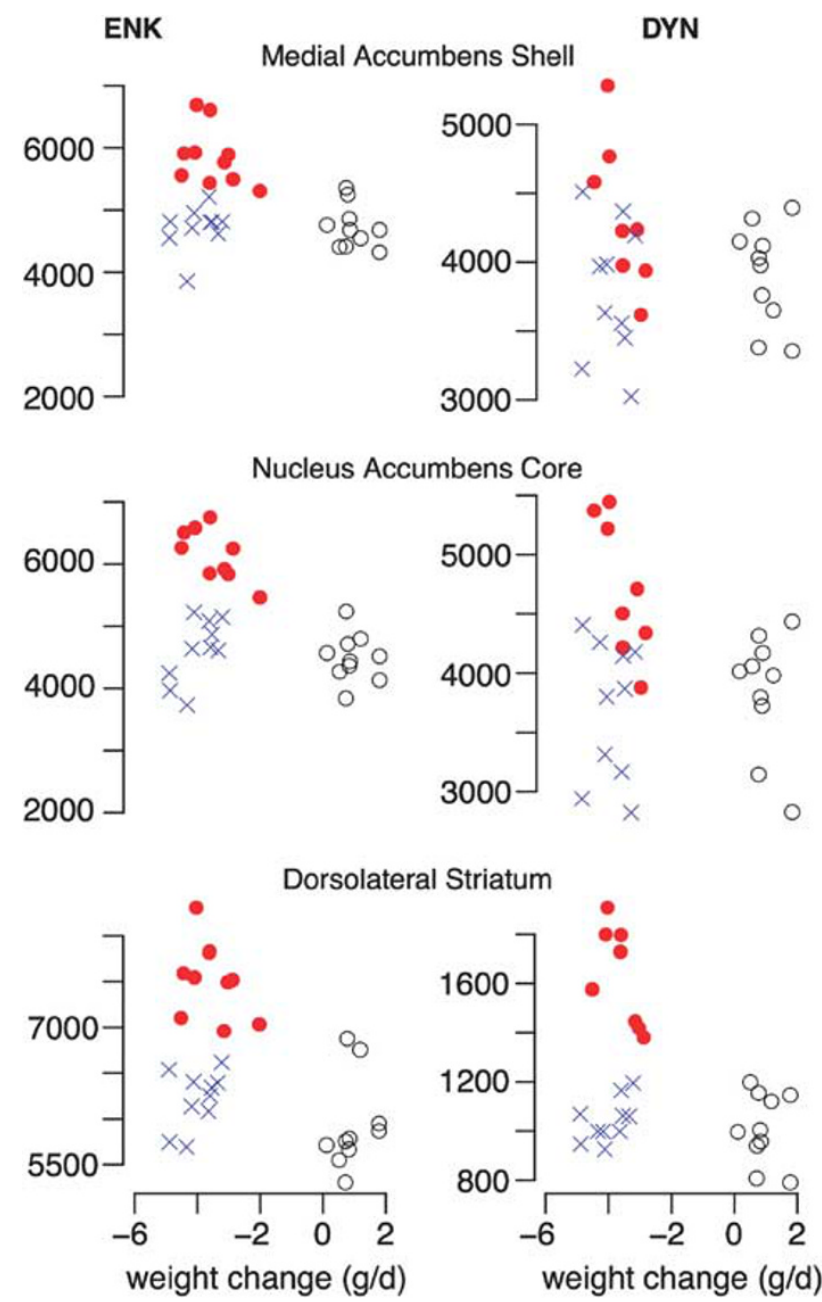

Figure 3 Scatterplots displaying gene expression levels $x$ weight change for ENK and DYN in experiment I, for individual subjects. The $Y$ axes show optical density (arbitrary units), an index of gene expression levels. $X$ axes show mean weight change over the second of two I0-day sleep restriction periods (see Materials and Methods for further details about the sleep deprivation protocol). Red circles, Sleep-Dep group; blue X's, Food-Restrict group; unfilled black circles, Home-Cage group.

arcuate nucleus, both sleep loss and food restriction altered transcriptional activity in parallel. The effects of sleep deprivation on striatal opioid systems, which are known to regulate palatable feeding, may help explain the 'junk-food snacking' seen with sleep curtailment in humans (Markwald et al, 2013; St-Onge et al, 2011; Weiss et al, 2010).

\section{Comparing the Effects of Sleep Deprivation and Food Deprivation}

The present results confirm prior work indicating that sleep loss in rodents engenders an endocrinological profile consistent with negative energy balance, in which rats lose weight and peripheral fat mass, and leptin levels decline along with concordant changes in feeding-regulatory peptide systems of the medial basal hypothalamus (Barf et al, 2012; Koban and Swinson, 2005; Koban et al, 2006; Newman et al, 2009; Rechtschaffen and Bergmann, 1995). In this sense, transcriptional changes in arcuate nucleus NPY and CART were not qualitatively different in sleep loss $v s$ food restriction. Indeed, the degree to which arcuate nucleus-localized NPY gene expression was upregulated, and arcuate-localized CART downregulated, was similar between the two manipulations.

In numerous striatal subregions, however, the sleep lossassociated upregulation of ENK and DYN gene expression was not seen in Food-Restrict rats that lost the same amount of weight or in App-Control rats subjected to sleep deprivation stimuli during waking. Striatal ENK is modulated by motivational contingencies such as the expectation of scheduled feeding or contextual stimuli associated with palatable food, but not by food restriction (Schlitz et al, 2007; Will et al, 2007), and stimulation of striatal $\mu$-opioid receptors (the cognate receptors for ENK) strongly enhances palatable feeding and hedonic taste reactivity (Baldo and Kelley, 2007; Pecina and Berridge, 2005; Woolley et al, 2006; Zhang et al, 1998; Zhang and Kelley, 2002). A recent study showed that surges of endogenously released striatal ENK act as signals to overeat palatable foods (Difeliceantonio et al, 2012). Accordingly, with regard to food motivation, fluctuations in striatal ENK expression have been hypothesized to track short-term motivational contingencies associated with feeding (Kelley et al, 2005; Will et al, 2007). This model proposes that striatal ENK downregulation is the 'off-switch' that signals recent completion of feeding. Importantly, ENK upregulation in Sleep-Dep rats was present despite the escalation of daily food intake, suggesting perhaps that sleep deprivation renders the striatal ENK system insensitive to negative feedback control (ie, the feeding 'switch' is stuck in the 'on' position). Striatal DYN expression was also upregulated in the Sleep-Dep rats, indicating that medium-spiny neurons are affected by sleep loss in both the 'direct' and 'indirect' striatal output pathways, for which DYN and ENK are cellular markers (Steiner and Gerfen, 1998). The upregulation of DYN further distinguishes the effects of food restriction and sleep deprivation; food restriction produces a small suppression of DYN expression in the Acb core (Haberny and Carr, 2005; Will et al, 2007; also see present work). Again, these observations support the idea that the upregulation of striatal opioid gene expression in the SleepDep rats was independent of their energy-balance state. Indeed, it is possible that shorter sleep deprivation periods may affect striatal opioid systems without changing hypothalamic energy-sensing systems, a question that merits further study. The current results show, however, that sleep loss affects the transcriptional control of a telencephalic reward system in a manner dissociable from weight loss per se. Finally, the fact that sleep loss produced effects on striatal opioid systems beyond those seen with food restriction or yoked, forced locomotion in the COWs would suggest that the transcriptional upregulation of opioid systems cannot be explained as a consequence of stress alone; nevertheless, it will be interesting in future studies to directly compare the present regimen of sleep loss with other types and intensities of laboratory stressors (eg, restraint, shock-paired contexts) with regard to opioid expression.

In contrast to striatal opioid systems, transcriptional changes in $\mathrm{H} / \mathrm{O}$ and $\mathrm{MCH}$ either did not occur or were inconsistent in this study. Although we partly replicated the 


\section{EXPERIMENT 1:}
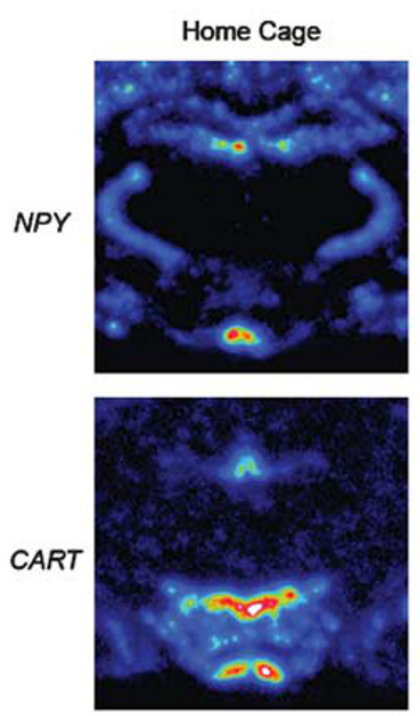

EXPERIMENT 2:
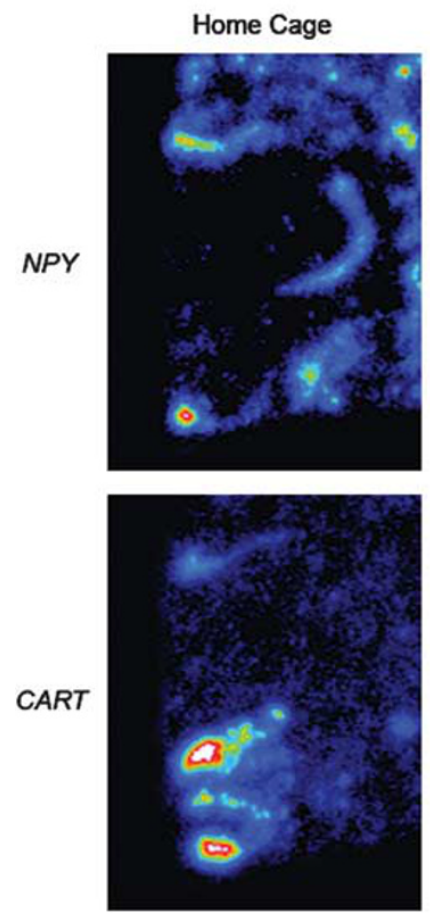

\section{NPY and CART}
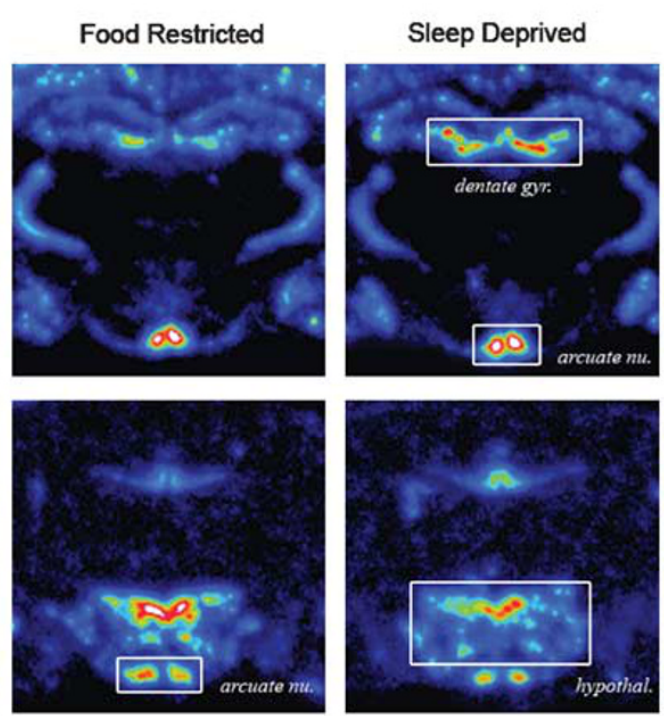

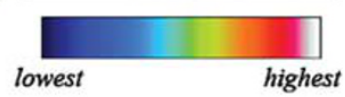

Sleep Deprived
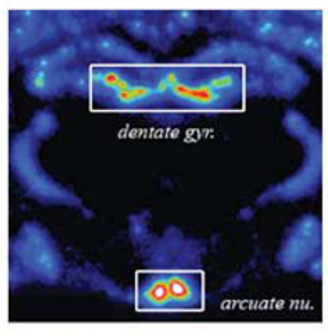

lowest
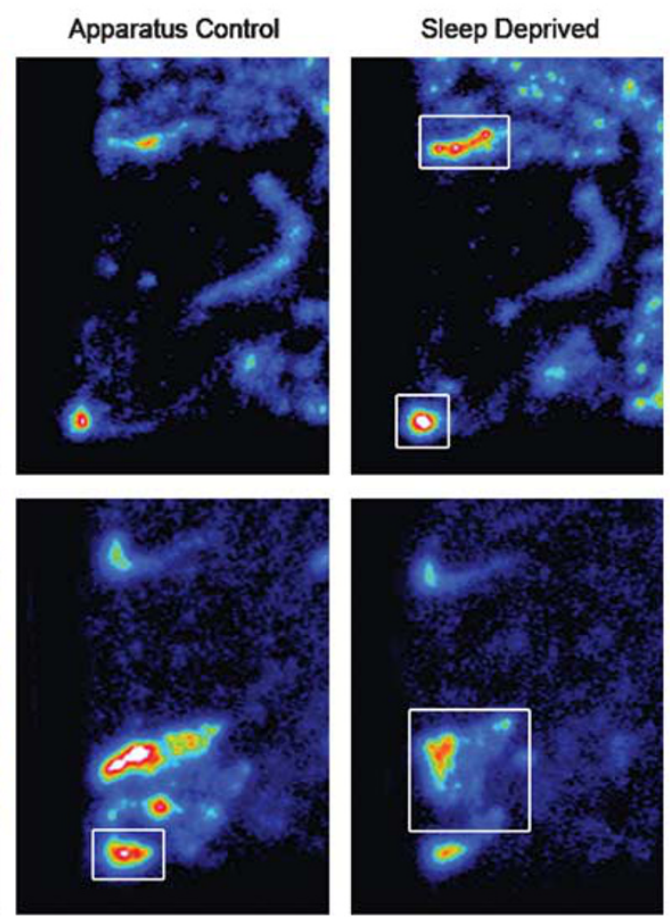

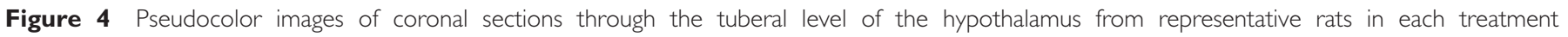

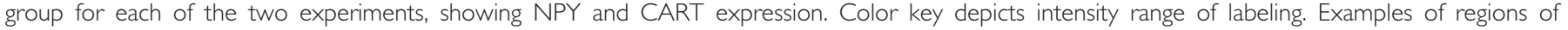
analysis for NPY in the hippocampal dentate gyrus and hypothalamic arcuate nucleus, and CART within and outside the arcuate nucleus, are shown with rectangles.

previously reported upregulation of H/O mRNA by sleep deprivation (Martins et al, 2010), the effect was inconsistent across the two experiments, suggesting that a global change in $\mathrm{H} / \mathrm{O}$ expression is not an obligatory accompaniment of feeding escalation in the present sleep deprivation paradigm.
Sleep Deprivation, NPY Expression, and Hippocampal Plasticity

Among the most selective of the transcriptional changes incurred by sleep loss was the robust upregulation of NPY expression in allo- and neocortex. Neocortical NPY is 

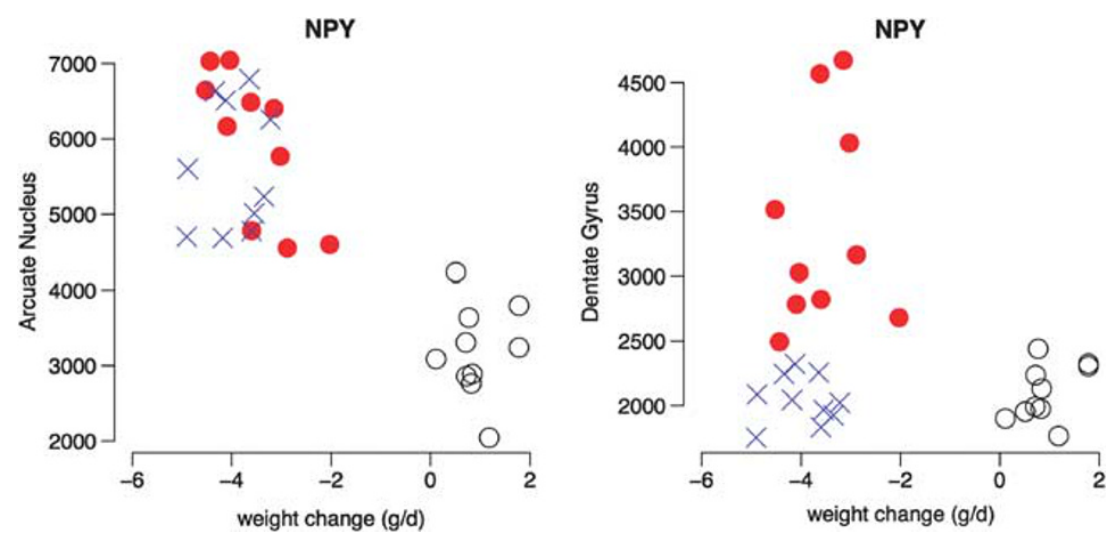

Figure 5 Scatterplots displaying gene expression levels $\times$ weight change for NPY in experiment I, for individual subjects. Axes show optical density by weight change; see Figure 3 figure legend for further details. Red circles, Sleep-Dep group; blue X's, Food-Restrict group. Scores for the Home-Cage controls are shown with unfilled black circles.

localized in interneurons (Hendry et al, 1984; Karagiannis et al, 2009), and hippocampal NPY is found both in GABA interneurons (Sperk et al, 2007) and granule cells (McQuiston et al, 1996). The role of NPY in hippocampus is not fully understood; however, several studies suggest a role in cellular defenses against excitatory stress. For example, NPY gene expression is upregulated by metabotropic glutamate receptor stimulation (Schwartzer and Sperk, 1998) and NPY in the dentate gyrus inhibits glutamate release (Whittaker et al, 1999) has anticonvulsive actions (Patrylo et al, 1999). NPY also reduces depolarization-induced $\mathrm{Ca}^{2+}$ influx into dentate granule cells (McQuiston et al, 1996), which could be responsible for NPY-induced inhibition of perforant pathway LTP (Whittaker et al, 1999). Hippocampal NPY may thus serve a neuroprotective role during conditions of cellular stress, but at the same time inhibit neuroplasticity relevant to learning. Finally, NPY produces a strong neuroproliferative effect in the dentate gyrus (Howell et al, 2005; Howell et al, 2007), which may represent a line of defense against the suppression of neurogenesis seen in sleep loss (GuzmanMarin et al, 2003). Further studies are needed to test these possibilities. In a general sense, however, the transcriptional regulation of dentate gyrus-NPY seen here may have implications for understanding sleep loss-induced changes in hippocampal-based learning and cognition.

\section{Clinical Implications}

Perhaps the clearest clinical implication of opioid upregulation engendered by sleep deprivation relates to understanding the increased preference for and 'snacking' on palatable fat- or carbohydrate-enriched foods (Markwald et al, 2013; St-Onge et al, 2011; Weiss et al, 2010), which appears to be a crucial casual factor for positive energy balance in human sleep loss (Markwald et al, 2013). In fact, recent results indicate that this 'snacking' is not entirely explained by changes in peripheral hunger- or satietymodulating hormones (Markwald et al, 2013), implicating central reward-modulatory systems such as the ENK system identified here. Furthermore, poor sleep is associated with reduced dietary restraint (Markwald et al, 2013) and binge eating (Trace et al, 2012), both of which may stem from altered central opioid function; for example, central $\mu$-opioid signaling is implicated in food bingeing (Nathan and Bullmnore, 2009). Similarly, striatal ENK changes could also contribute to bingeing in specific disorders such as night eating syndrome and sleep-related eating disorder, both of which are characterized by sleep disruption (Vinai et al, 2012).

Another important consideration is that striatal ENK and DYN were upregulated in concert. DYN signaling is associated with aversive or depression-like effects in animal studies (Nestler and Carlezon, 2006), and striatal DYN levels are elevated in post-mortem brains of suicide victims and in animal models of stress and drug withdrawal (Horner et al, 2009; Hurd et al, 1997; Shippenberg et al, 2007; Shirayama et al, 2004). Hence, coordinated upregulation of ENK and DYN could produce a mixture of depressed mood and increased preference for palatable food, as is often observed in eating disorders characterized by dysfunctional 'emotional eating.' A related possibility is that sleep deprivationinduced upregulation of ENK could have a normalizing influence on mood in pathological states associated with already-elevated DYN levels. A specific case could be the palliative effect of acute sleep deprivation in depression (Benedetti and Colombo, 2010; Giedke and Schwarzler, 2002; Gillin et al, 2001; Wu et al, 2009). Although speculative, these hypotheses lead to testable predictions, particularly with the advent of PET imaging techniques capable of detecting in vivo opioid signaling.

\section{FUNDING AND DISCLOSURE}

The authors declare that over the past three years RB has received compensation from Sanofi-Aventis, Merck, the American Academy of Sleep Medicine and the Sleep Research Society. The remaining authors declare no conflict of interest.

\section{ACKNOWLEDGEMENTS}

This paper is dedicated to the memory of Dr Ann E Kelley. This work was supported by NHLBI grant R01 HL086465 to RMB. BAB was also supported by NIMH grant R01 MH 074723. 


\section{REFERENCES}

Baldo BA, Kelley AE (2007). Discrete neurochemical coding of distinguishable motivational processes: insights from nucleus accumbens control of feeding. Psychopharmacology (Berl) 191: 439-459.

Barf RP, Van Dijk G, Scheurink AJ, Hoffmann K, Novati A, Hulshof $\mathrm{HJ}$ et al (2012). Metabolic consequences of chronic sleep restriction in rats: changes in body weight regulation and energy expenditure. Physiol Behav 107: 322-328.

Benedetti F, Colombo C (2010). Sleep deprivation in mood disorders. Neuropsychobiology 64: 141-151.

Cappuccio FP, Taggart FM, Kandala NB, Currie A, Peile E, Stranges $S$ et al (2008). Meta-analysis of short sleep duration and obesity in children and adults. Sleep 31: 619-626.

Difeliceantonio AG, Mabrouk OS, Kennedy RT, Berridge KC (2012). Enkephalin surges in dorsal neostriatum as a signal to eat. Curr Biol 22: 1918-1924.

Giedke H, Schwarzler F (2002). Therapeutic use of sleep deprivation in depression. Sleep Med Rev 6: 361-377.

Gillin JC, Buchsbaum M, Wu J, Clark C, Bunney W Jr (2001). Sleep deprivation as a model experimental antidepressant treatment: findings from functional brain imaging. Depress Anxiety 14: 37-49.

Guzman-Marin R, Suntsova N, Stewart DR, Gong H, Szymusiak R, McGinty D (2003). Sleep deprivation reduces proliferation of cells in the dentate gyrus of the hippocampus in rats. J Physiol 549: 563-571.

Haberny SL, Carr KD (2005). Comparison of basal and D-1 dopamine receptor agonist-stimulated neuropeptide gene expression in caudate-putamen and nucleus accumbens of ad libitum fed and food-restricted rats. Brain Res Mol Brain Res 141: 121-127.

Hanlon EC, Van Cauter E (2011). Quantification of sleep behavior and of its impact on the cross-talk between the brain and peripheral metabolism. Proc Natl Acad Sci USA 108(Suppl 3): 15609-15616.

Hendry SH, Jones EG, DeFelipe J, Schmechel D, Brandon C, Emson PC (1984). Neuropeptide-containing neurons of the cerebral cortex are also GABAergic. Proc Natl Acad Sci USA 81: 6526-6530.

Hillebrand JJG, de Wied D, Adan RAH (2002). Neuropeptides, food intake and body weight regulation: a hypothalamic focus. Peptides 23: 2203-2306.

Horner KA, Noble ES, Lauterbach EC (2009). Differential regulation of prodynophin, $\mathrm{c}$-fos, and serotonin transporter mRNA following withdrawal from a chronic, escalating dose regimen of D-amphetamine. Synapse 63: 257-268.

Howell OW, Doyle K, Goodman JH, Scharfman HE, Herzog H, Pringle A et al (2005). Neuropeptide Y stimulates neuronal precursor proliferation in the post-natal and adult dentate gyrus. J Neurochem 93: 560-570.

Howell OW, Silva S, Scharfman HE, Sosunov AA, Zaben M, Shatya A et al (2007). Neuropeptide $\mathrm{Y}$ is important for basal and seizure-induced precursor cell proliferation in the hippocampus. Neurobiol Dis 26: 174-188.

Hurd YL, Herman MM, Hyde TM, Bigelow LB, Weinberger DR, Kleinman JE (1997). Prodynorphin mRNA expression is increased in the patch vs matrix compartment of the caudate nucleus in suicide subjects. Mol Psychiatry 2: 495-500.

Karagiannis A, Gallopin T, David C, Battaglia D, Geoffroy H, Rossier J et al (2009). Classification of NPY-expressing neocortical interneurons. J Neurosci 29: 3642-3659.

Kelley AE, Baldo BA, Pratt WE (2005). A proposed hypothalamicthalamic-striatal axis for the integration of energy balance, arousal, and food reward. J Comp Neurol 493: 72-85.

Koban M, Le WW, Hoffman GE (2006). Changes in hypothalamic corticotropin-releasing hormone, neuropeptide $\mathrm{Y}$, and proopiomelanocortin gene expression during chronic rapid eye movement sleep deprivation of rats. Endocrinology 147: 421-431.

Koban M, Swinson KL (2005). Chronic REM-sleep deprivation of rats elevates metabolic rate and increases UCP1 gene expression in brown adipose tissue. Am J Physiol Endocrinol Metab 289: E68-E74.

Markwald RR, Melanson EL, Smith MR, Higgins J, Perreault L, Eckel RH et al (2013). Impact of insufficient sleep on total energy expenditure, food intake, and weight gain. Proc Natl Acad Sci USA 110: 5695-5700.

Martins PJ, Marques MS, Tufik S, D’Almeida V (2010). Orexin activation precedes increased NPY expression, hyperphagia, and metabolic changes in response to sleep deprivation. Am J Physiol Endocrinol Metab 298: E726-E734.

McQuiston AR, Petrozzino JJ, Connor JA, Colmers WF (1996). Neuropeptide Y1 receptors inhibit N-type calcium currents and reduce transient calcium increases in rat dentate granule cells. J Neurosci 16: 1422-1429.

Nathan PJ, Bullmnore ET (2009). From taste hedonics to motivational drive: central mu-opioid receptors and bingeeating behavior. Int J Neuropsychopharmacol 12: 995-1008.

Nedeltcheva AV, Kessler L, Imperial J, Penev PD (2009). Exposure to recurrent sleep restriction in the setting of high caloric intake and physical inactivity results in increased insulin resistance and reduced glucose tolerance. J Clin Endocrinol Metab 94: 3242-3250.

Nestler EJ, Carlezon WA Jr. (2006). The mesolimbic reward circuit in depression. Biol Psychiatry 59: 1151-1159.

Newman SM, Paletz EM, Obermeyer WH, Benca RM (2009). Sleep deprivation in pigeons and rats using motion detection. Sleep 32: 1299-1312.

Patel SR, Hu FB (2008). Short sleep duration and weight gain: a systematic review. Obesity (Silver Spring) 16: 643-653.

Patrylo PR, van den Pol AN, Spencer DD, Williamson A (1999). NPY inhibits glutamatergic excitation in the epileptic human dentate gyrus. J Neurophysiol 82: 478-483.

Pecina S, Berridge KC (2005). Hedonic hot spot in nucleus accumbens shell: where do mu-opioids cause increased hedonic impact of sweetness? J Neurosci 25: 11777-11786.

Rechtschaffen A, Bergmann BM (1995). Sleep deprivation in the rat by the disk-over-water method. Behav Brain Res 69: 55-63.

Schiltz CA, Bremer QZ, Landry CF, Kelley AE (2007). Foodassociated cues alter forebrain functional connectivity as assessed with immediate early gene and proenkephalin expression. BMC Biol 5: 16.

Schwarzer C, Sperk G (1998). Glutamate-stimulated neuropeptide Y mRNA expression in the rat dentate gyrus: a prominent role of metabotropic glutamate receptors. Hippocampus 8: 274-288.

Shippenberg TS, Zapata A, Chefer VI (2007). Dynorphin and the pathophysiology of drug addiction. Pharmacol Ther 116: 306-321.

Shirayama Y, Ishida H, Iwata M, Hazama GI, Kawahara R, Duman RS (2004). Stress increases dynorphin immunoreactivity in limbic brain regions and dynorphin antagonism produces antidepressant-like effects. J Neurochem 90: 1258-1268.

Sperk G, Hamilton T, Colmers WF (2007). Neuropeptide Y in the dentate gyrus. Prog Brain Res 163: 285-297.

Spiegel K, Leproult R, L'Hermite-Baleriaux M, Copinschi G, Penev PD, Van Cauter E (2004b). Leptin levels are dependent on sleep duration: relationships with sympathovagal balance, carbohydrate regulation, cortisol, and thyrotropin. J Clin Endocrinol Metab 89: 5762-5771.

Spiegel K, Tasali E, Penev P, Van Cauter E (2004a). Brief communication: sleep curtailment in healthy young men is associated with decreased leptin levels, elevated ghrelin levels, and increased hunger and appetite. Ann Intern Med 141: $846-850$. 
St-Onge MP, Roberts AL, Chen J, Kelleman M, O'Keeffe M, RoyChoudhury A et al (2011). Short sleep duration increases energy intakes but does not change energy expenditure in normal-weight individuals. Am J Clin Nutr 94: 410-416.

Steiner H, Gerfen CR (1998). Role of dynorphin and enkephalin in the regulation of striatal output pathways and behavior. Exp Brain Res 123: 60-76.

Trace SE, Thornton LM, Runfola CD, Lichtenstein P, Pedersen NL, Bulik CM (2012). Sleep problems are associated with binge eating in women. Int J Eat Disord 45: 695-703.

Van Cauter E, Knutson KL (2008). Sleep and the epidemic of obesity in children and adults. Eur J Endocrinol 159(Suppl 1): S59-S66.

Vinai P, Ferri R, Ferini-Strambi L, Cardetti S, Anelli M, Vallauri P et al (2012). Defining the borders between sleep-related eating disorder and night eating syndrome. Sleep Med 13: 686-690.

Weiss A, Xu F, Storfer-Isser A, Thomas A, Ievers-Landis CE, Redline S (2010). The association of sleep duration with adolescents' fat and carbohydrate consumption. Sleep 33: 1201-1209.
Whittaker E, Vereker E, Lynch MA (1999). Neuropeptide Y inhibits glutamate release and long-term potentiation in rat dentate gyrus. Brain Res 827: 229-233.

Will MJ, Vanderheyden WM, Kelley AE (2007). Striatal opioid peptide gene expression differentially tracks short-term satiety but does not vary with negative energy balance in a manner opposite to hypothalamic NPY. Am J Physiol Regul Integr Comp Physiol 292: R217-R226.

Woolley JD, Lee BS, Fields HL (2006). Nucleus accumbens opioids regulate flavor-based preferences in food consumption. Neuroscience 143: 309-317.

Wu JC, Kelsoe JR, Schachat C, Bunney BG, DeModena A, Golshan S et al (2009). Rapid and sustained antidepressant response with sleep deprivation and chronotherapy in bipolar disorder. Biol Psychiatry 66: 298-301.

Zhang M, Gosnell BA, Kelley AE (1998). Intake of high-fat food is selectively enhanced by mu opioid receptor stimulation within the nucleus accumbens. J Pharmacol Exp Ther 285: 908-914.

Zhang M, Kelley AE (2002). Intake of saccharin, salt, and ethanol solutions is increased by infusion of a mu opioid agonist into the nucleus accumbens. Psychopharmacology (Berl) 159: 415-423.

Supplementary Information accompanies the paper on the Neuropsychopharmacology website (http://www.nature.com/npp) 